\title{
Feedback Effects at Stable and Unstable Resonant External Cavity Lengths
}

\author{
A. S. van de Nes, J. J. H. B. Schleipen, G. W. 't Hooft, and M. P. van Exter
}

\begin{abstract}
External feedback applied to a high-power semiconductor laser is found to be responsible for a drop in output power and an increased noise-level when the optical external cavity length is an integral multiple of the optical internal cavity length. The power drops and extra noise originates from a scan of the quasisingle-mode laser over its internal cavity modes, caused by a lack of interferometric stability. In the case of interferometric stability, the wavelength of the laser exhibits a staircase-like dependence on the external cavity length. All experimental results can be described by a simple rate equation model.
\end{abstract}

Index Terms-Intensity noise, laser modes, optical feedback, semiconductor laser.

\section{INTRODUCTION}

I $\mathrm{N}$ OPTICAL recorders, it is practically unavoidable that part of the light reflected from the disk reenters the laser source. With the introduction of the next-generation optical recorders, with new light paths and different semiconductor lasers, the importance of feedback has to be reviewed. Generally, the induced effects are related to the amount of optical feedback, as described in [1]. For the operation of optical recorders, the most inconvenient consequence of optical feedback is increased intensity noise. This is one of the subjects studied in this paper.

We have studied a 30-mW high-power AlGaInP semiconductor laser (Mitsubishi ML1016R-01) emitting light at a wavelength of $661 \mathrm{~nm}$. A schematic overview of the experimental setup is shown in Fig. 1. A mirror attached to a piezo is placed on a translation stage to provide the optical feedback for a selectable external cavity length. Two lenses are used to collimate the light emitted by the laser and focus it onto the feedback mirror. In between the two lenses, a beam splitter has been inserted to deflect part of the light through an optical Faraday isolator to the detection setup, where we can simultaneously measure the optical output power and the emission wavelength. A homemade photodetector was connected to a multimeter (Keithley 2000) to measure the average optical output power of the laser; the fluctuations in the output power are displayed on a spectrum analyzer (HP8515E). The emission wavelength of the light is measured with a monochromator, consisting of a 1200 lines/mm grating and two concave mirrors with a focal length of $1.25 \mathrm{~m}$, yielding a resolution better than $0.17 \AA$.

Manuscript received November 20, 2001; revised May 6, 2002.

A. S. van de Nes is with Delft University of Technology, Delft, The Netherlands (e-mail: A.vandeNes@tnw.TUDelft.nl).

J. J. H. B. Schleipen and G. W. 't Hooft are with Philips Research Laboratories, Eindhoven, The Netherlands.

M. P. van Exter is with University of Leiden, Leiden, The Netherlands.

Publisher Item Identifier 10.1109/JQE.2002.801033.

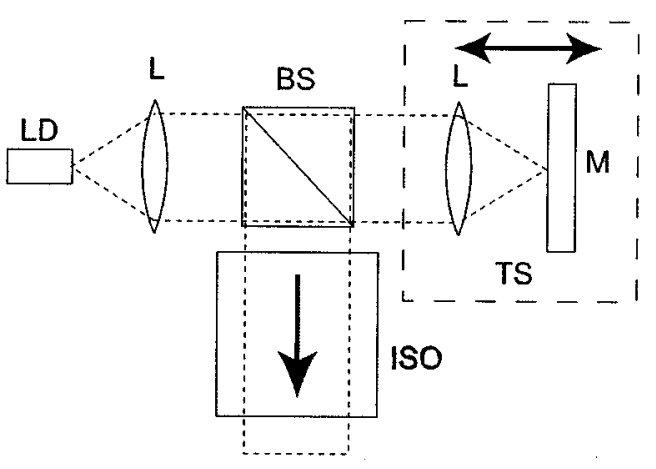

Fig. 1. Experimental setup. LD: laser diode. L: lens. BS: beam splitter. TS: translation stage. ISO: optical isolator.

\section{THE EXPERIMENT}

A measurement of the output power as a function of the laser current yields a threshold current of $46.8 \mathrm{~mA}$. The longitudinal mode spacing was measured to be $0.80 \AA$, yielding an optical internal cavity length of 2.71(2) $\mathrm{mm}$. The modulation transfer function has been measured as a function of the laser current. The derived relaxation oscillation frequency and damping as a function of the pump parameter can be used to obtain an estimate of the cavity loss rate $\Gamma_{c} \approx 2 \cdot 10^{11} \mathrm{~s}^{-1}$. From this point on, we will omit the term optical when discussing optical cavity lengths.

The amount of optical feedback cannot be measured directly. Based on the transmission of the optical elements, up to $10 \%$ of the emitted light might be fed back into the laser. However, this value has to be corrected for the imperfect mode overlap by the limited numerical aperture (0.22) of the collimating lens, and the alignment of the optics. When optical feedback is applied to the laser, the reduction of $1 \mathrm{~mA}$ in the laser threshold current can be used to estimate the feedback intensity ratio to be $10^{-4}$, as described below. Measurements with a Fabry-Pérot interferometer showed that the laser generally operated in a single internal cavity mode. As we have seen, no sign of coherence collapse, we expect our laser to be operating in regime III as defined in [1]. Practically all experimental and theoretical work on optical feedback is concentrated on lasers that operate in a single longitudinal mode, whereas we are amongst the few who explicitly consider the possibility of quasi single-mode operation, i.e., single-mode operation in several different longitudinal modes.

Fig. 2(a) shows the output power as a function of small changes in the interferometrically stable external cavity length on top of a length offset of 62.0(2) mm. The current applied to the semiconductor laser is $55 \mathrm{~mA}$. The solid curve shows that the output power is a recurrent signal with a period of 


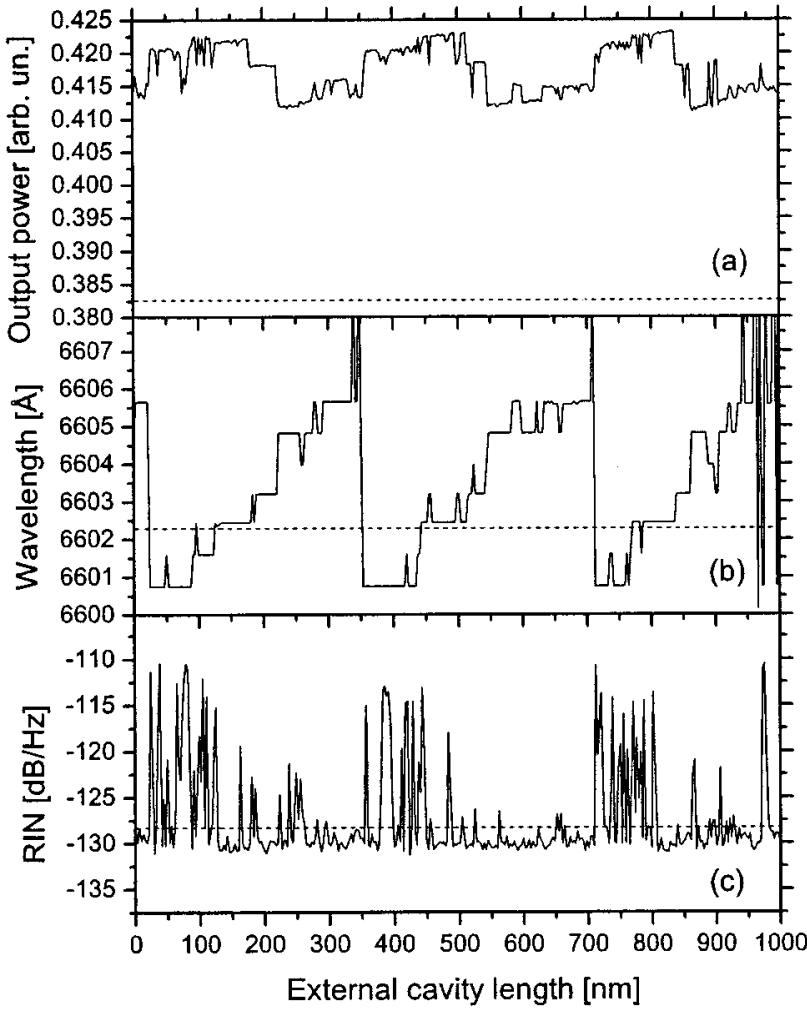

Fig. 2. As a function of the external cavity length we plot: (a) the optical output power of the laser; (b) the emission wavelength of the lasing mode; and (c) the RIN. Offset of the external cavity length: $62.0(2) \mathrm{mm}$. Current applied to the laser: $55 \mathrm{~mA}$. Experiments with and without optical feedback are represented by solid curves and dotted lines, respectively.

$\lambda / 2=330 \mathrm{~nm}$ and has a value that is always larger than the output power level without optical feedback (dotted line). The output power is not a neat sinusoidal signal, as might be expected for a single longitudinal mode, but is somewhat erratic instead, because there are several longitudinal modes involved in the quasi single-mode lasing process (as explained below).

Fig. 2(b) shows the wavelength of the dominant longitudinal mode as a function of the external cavity length. Note that the height of each discrete step is an integer times the longitudinal mode spacing of $0.80 \AA$. Each different emission wavelength is related to lasing in a different longitudinal mode. After a step to a different longitudinal mode, the laser is operating in a single mode again; therefore we will call the laser quasi single-mode. The dotted line represents the wavelength of the laser without optical feedback.

To understand why the laser exhibits longitudinal mode hops when the external cavity length is fine tuned over a sub wavelength interval, we recall that the phase relation between the feedback and intra-cavity light determines whether a mode is enhanced or reduced. The selection of the preferred lasing mode can now be understood easily, by comparing the (de-)amplification of the longitudinal modes in the frequency domain with the overlay of two comb-shaped net gain curves with a different tooth period (see Fig. 3). The first comb represents the longitudinal internal cavity modes with a spacing of approximately $55 \mathrm{GHz}$; the other combs represent the external cavity modes with a spacing of $c /\left(2 L_{e}\right) \approx 2.4 \mathrm{GHz}$, where $c$ is the speed

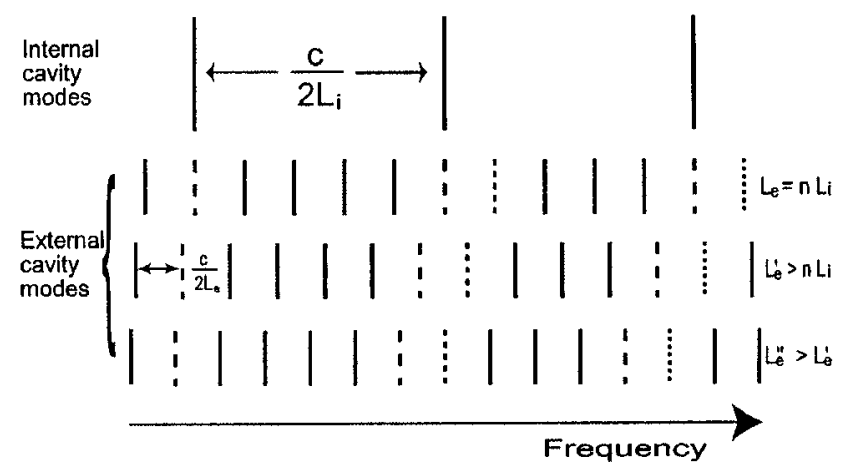

Fig. 3. Schematic view of the frequency of the internal and external cavity modes represented by large and small lines, respectively. External cavity mode spacing decreases with an increasing external cavity length $\left(L_{e}\right)$.

of light and $L_{e}$ is the external cavity length. If the external cavity length approximately equals an integral multiple of the internal cavity length, the external cavity modes will simultaneously overlap with all internal cavity modes, to simultaneously favor all modes, or for a $\lambda / 4$ different length simultaneously fall in between the internal cavity modes. For other lengths, the external cavity modes will not simultaneously overlap with the internal cavity modes, and the amount of overlap, as given by the exact external cavity length, will codetermine which longitudinal mode will be the preferred lasing mode.

Fig. 2(c) shows the relative intensity noise (RIN), in the frequency range of $1-3 \mathrm{MHz}$, as a function of the external cavity length, simultaneously measured with the output power and wavelength. Noise bursts originate from mode jumps between two or more almost equally preferred longitudinal modes. The density of noise bursts is highest, and the mode competition is apparently strongest, when the "wavelength-staircase" is at its lowest step. Here, several modes must be almost equal in strength and compete for lasing operation. The dotted line shows the RIN level without optical feedback. It is slightly higher than the lowest RIN levels observed with optical feedback, which was expected as an increase in output power should result in a lower RIN level.

The exact shape of the "wavelength-staircase" depends on the external cavity length, or more specifically, on the ratio of external and internal cavity lengths. In Fig. 2, the external cavity length is 22.8 times the internal cavity length. Fig. 4(a) shows the experimental results (still at a laser current of $55 \mathrm{~mA}$ ) for four different ratios: 22.7, 22.9, 23.1, and 23.3. Note that the shape of the "wavelength-staircase" is mirrored at an integer ratio.

For an external cavity length close to such an integral multiple of the internal cavity length, the observed wavelength scan is easy to understand in the frequency domain, as shown in Fig. 3. If the external cavity length is slightly larger than an integral multiple, the spacing between the external cavity modes becomes smaller. A subwavelength increase in cavity length and, consequently, a decrease in external cavity frequency, will then shift the frequency overlap (or resonance condition) from one internal cavity mode to its higher frequency neighbor. The resulting wavelength scan is, thus, expected to resemble a descending staircase, as is indeed observed experimentally. For ex- 


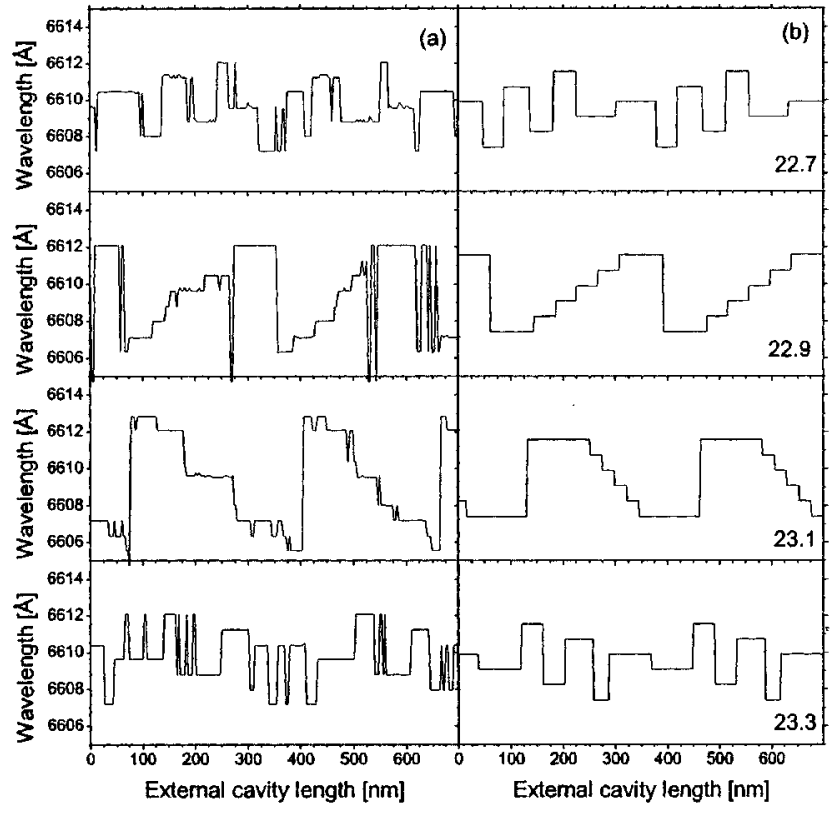

Fig. 4. Emission wavelength of the dominant longitudinal mode as a function of the external cavity length as: (a) measured and (b) simulated numerically. From top to bottom, the ratio of the external cavity length and the internal cavity length was 22.7, 22.9, 23.1, and 23.3.

ternal cavity lengths further away from an integral multiple, the steepness of the "wavelength-staircase" will decrease. For even larger external cavity lengths, the shape of the staircase is much harder to predict and yields, for example, the top and bottom graphs in Fig. 4(a).

We have also performed a numerical study of the rate equations for the carrier density $N$, the photon density per mode $S_{m}$, and the corresponding oscillation frequency $\omega_{m}$ of that mode. Based on Fig. 4(a), we estimate the number of relevant longitudinal modes to be 7 , resulting in a total of 15 coupled equations to simulate. The equations that we have used are similar to those described in [2] and [3], where optical feedback has been incorporated

$$
\begin{aligned}
\frac{d N}{d t} & =J-\Gamma_{\mathrm{sp}} N-\sum_{m} \Gamma_{\mathrm{st}} S_{m} \\
\frac{d S_{m}}{d t} & =\Gamma \Gamma_{\mathrm{st}} S_{m}-\Gamma_{c} S_{m}+\beta \Gamma \Gamma_{\mathrm{sp}} N \\
\frac{d \phi_{m}}{d t} & =\omega_{m}-\frac{2 \pi c}{2 L_{i} \mu}\left(m-\phi_{\mathrm{fb}}\right)
\end{aligned}
$$

where

$\begin{array}{ll}J & \text { effective injected current density; } \\ \Gamma_{\mathrm{sp}} & \text { spontaneous emission rate; } \\ \Gamma_{\mathrm{st}} & \text { stimulated emission rate; } \\ \Gamma_{c} & \text { cavity loss rate; } \\ \Gamma & \text { confinement factor; } \\ \beta & \text { spontaneous emission factor; } \\ c & \text { speed of light; } \\ \mu L_{i} & \text { optical internal cavity length; } \\ m & \text { mode number; } \\ \phi_{\mathrm{fb}} & \text { phase change caused by the optical feedback. }\end{array}$

The stimulated emission rate $\Gamma_{\mathrm{st}}$ and the refractive index $\mu$ both depend on the carrier density, the photon density, and the oscillation frequency. In the stimulated emission rate, we have incorporated the spectral response, the correction for gain compression at high photon densities, and linearized it around the carrier density at transparency. The cavity loss rate $\Gamma_{c}=(c / \mu)\left(\alpha_{s}+\alpha_{m}+\alpha_{\mathrm{fb}}\right)$ depends on the scatter losses $\alpha_{s}$, the mirror losses $\alpha_{m}$, and the loss or gain caused by the optical feedback

$$
\begin{aligned}
\alpha_{\mathrm{fb}} & =\frac{1}{2 L_{i}} \ln \frac{1}{1+\kappa^{2}-2 \kappa \cos \omega_{m} \tau} \\
\phi_{\mathrm{fb}} & =\arctan \frac{\kappa \sin \omega_{m} \tau}{1-\kappa \cos \omega_{m} \tau}
\end{aligned}
$$

where $\kappa=\left(1-R_{2}\right) \sqrt{R_{3} / R_{2}}, R_{2}$ is the reflection coefficient of the exit facet, $R_{3}$ is the effective reflection coefficient of the external feedback mirror, and $\tau$ is the external cavity round trip time. As the feedback intensity ratio in our experiment was low, we have neglected multiple reflections within the external cavity.

Combining (1) and (2) for the steady-state situation at lasing threshold and neglecting the spontaneous emission term results in $\Delta I_{\mathrm{th}} / I_{\mathrm{th}}=\Delta \Gamma_{c} / \Gamma_{c}=c \alpha_{\mathrm{fb}} / \mu \Gamma_{c}$. We can use the cavity loss rate without optical feedback $\Gamma_{c} \approx 2 \cdot 10^{11} \mathrm{~s}^{-1}$, the measured internal cavity length $\mu L_{i}=2.7 \cdot 10^{-3} \mathrm{~m}$, the speed of light $c=3.0 \cdot 10^{8} \mathrm{~ms}^{-1}$, the injection current at threshold $I_{\mathrm{th}}=46.8 \mathrm{~mA}$, the injection current shift caused by the optical feedback $\Delta I_{\mathrm{th}}=-1 \mathrm{~mA}$, and an estimate of the reflectivity of the exit facet $\left(R_{2} \approx 0.05\right)$ to obtain a rough estimate of the effective reflectivity of our feedback mirror $R_{3} \approx 1 \cdot 10^{-4}$.

For a variable external cavity length, the time dynamics has been studied over a period of $15 \mathrm{~ns}$, using a fourth-order Runge-Kutta solving algorithm. After this period, a steady-state situation has been reached. Fig. 4(b) shows the numerical results for the emission wavelength of the dominant longitudinal mode, as a function of the external cavity length and for several external cavity length offsets. These results can be directly compared to the experimental data plotted in Fig. 4(a). The same "wavelength-staircase" is observed in the experimental results as in the numerical results. The perfect agreement demonstrates the quality of the measurements and the validity of the theoretical model.

\section{AVERAGING}

For a commercial optical recorder, the interferometric stability used up to now is not readily reached. In optical recorders, the distance from the laser to the spinning disc is less stable and typically varies over a range of several wavelengths. To simulate this variation in the experiment, we have applied a $100-\mathrm{Hz}$ sinusoidal signal to the piezo to modulate the external cavity length over a range of a few wavelengths, effectively averaging over several longitudinal mode configurations. We will refer to the external cavity length, the output power, and intensity noise in the case of such a scan as the unstable external cavity length, the averaged power, and the averaged intensity noise.

Fig. 5(a) shows the averaged power as a function of the unstable external cavity length for a current of $52.5 \mathrm{~mA}$ applied 


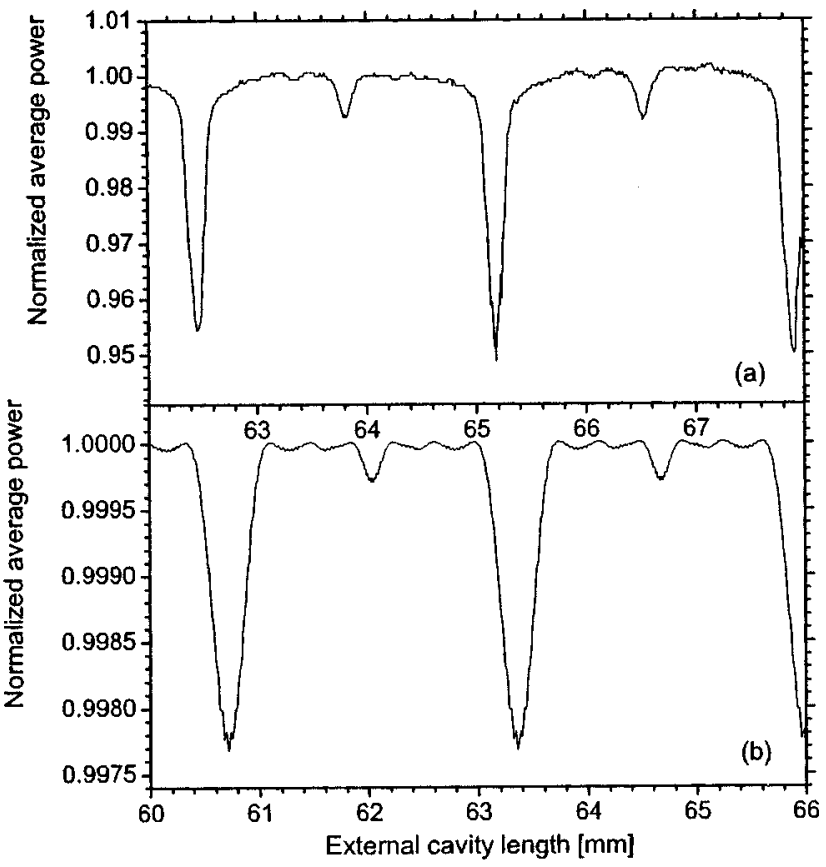

Fig. 5. Normalized averaged output power as a function of the unstable, i.e., actively modulated, external cavity length: (a) as measured at a laser current of $52.5 \mathrm{~mA}$ and (b) as simulated. The drops in the output power occur when the external cavity length equals an integral multiple of the internal cavity length. Note the qualitative agreement for both large and small power drops.

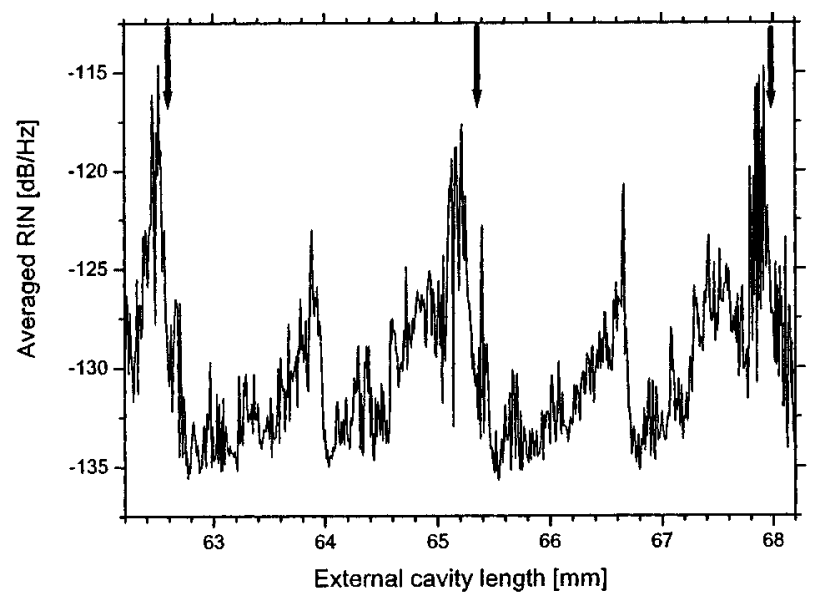

Fig. 6. Averaged relative intensity noise as a function of the unstable external cavity length. Noise bursts occur at integral multiples of the internal cavity length (indicated by arrows).

to the laser. Large drops in the averaged power are found at integral multiples of the internal cavity length. In between these large drops, several smaller drops are found at integer fractions of the internal cavity length. The drops in average power can be understood by first considering the power change as a function of the stable external cavity length. When $L_{e}$ is equal to an integral multiple of the internal cavity length, all modes are in- or out-of-phase at the same time and the output power depends crucially on the exact cavity length. For stable external cavity lengths different from an integer times the internal cavity length, the internal and external cavity modes will be detuned from each other, and the laser will operate at the strongest mode possible for that configuration. Therefore, while switching from optimum mode to optimum mode during the piezo scan, the averaged power can effectively be higher than for the case of an unstable external cavity length equal to an exact integral multiple. The smaller drops at an integer fraction of the internal cavity length have a similar origin but the effect is less dramatic. The spacing of the large power drops can directly be used to acquire an estimate of the (optical) internal cavity length, yielding $2.716(2) \mathrm{mm}$.

The averaged power as a function of the unstable external cavity length has been studied numerically too. The simulated output power is averaged over a few wavelengths to account for the piezo scan; the results are plotted in Fig. 5(b). The large drops at integral multiples of the internal cavity length are reproduced, and even the smaller drops can be observed in the numerical results. Note that the internal cavity length in the simulated results was $2.64 \mathrm{~mm}$ instead of $2.72 \mathrm{~mm}$, which explains the 2-mm offset between both plots. We are satisfied with the qualitative agreement and have not optimized all parameters in view of the time consuming calculations.

Fig. 6 shows the relative intensity noise as a function of the unstable external cavity length at a current of $55 \mathrm{~mA}$ and again plotted over a range of $6 \mathrm{~mm}$, i.e., 2.2 times the internal cavity length. Averaged noise bursts are visible at multiples of the internal cavity length (indicated by arrows), and smaller bursts can be observed at integer fractions. The explanation is the same as before: at these integer fractions, the mode competition is strongest as several longitudinal modes are equally favored.

The drops in the average power reported in this paper are similar to those reported in [4] and [5], where the interferometric stability was destroyed by a current modulation of $1 \mathrm{~mA}$. However, their theoretical description of the output power as a function of the external cavity length is different from the one presented here. Quite surprisingly, it even yields power peaks instead of power drops at external cavity lengths equal to an integer times the internal cavity length. Note that we are certain that the drops in the average output power really occur at integral multiples, as the offset of the external cavity length was carefully measured and agreement with the numerical results is very good. The reason for this discrepancy is tha,t in [4], the feedback phase was assumed to be an exact multiple of $2 \pi$ for the two internal cavity modes considered in that paper, whereas we explicitly average over a variation of a few wavelengths in the external cavity length to account for the lack of interferometric stability. The absence of the smaller peaks in the description of [4] is caused by the restriction to only two modes. When more internal cavity modes are considered, smaller peaks at integer internal cavity length fractions should also appear.

The experiments have been repeated for different values of the injection current and the amount of optical feedback. The general shape of the results is similar to the description given above. The power drop is proportional to the amount of optical feedback and the injected current. For higher injection currents, the amount of noise bursts decreases. This can be explained by a corresponding decrease of the linewidth of the lasing mode, which co-determines the width of the region of spectral overlap that fulfills both the mode selection criterion of the internal and external cavity. 


\section{CONCLUSION}

In conclusion, we have studied a high-power semiconductor laser subject to optical feedback. We have simultaneously measured its laser output power, wavelength, and RIN as a function of the stable external cavity length. Within an external cavity length variation of $\lambda / 2$, these properties change considerably. Most noticeable is the scan of the wavelength over a series of internal cavity modes, which satisfy the resonance condition for in-phase feedback in a consecutive manner. For some external cavity lengths, the intensity noise increases significantly as a result of mode-hopping.

We have also measured the averaged output power and averaged RIN as a function of the unstable external cavity length, which is then averaged over a range of a few wavelengths. This is relevant for optical recorders, where the distance from laser to disc does not have interferometric stability. The averaged output power is observed to drop at unstable external cavity lengths that are equal to an integral multiple of the internal cavity length. In between two integral multiples, at rational fractions of the internal cavity length, smaller drops in the averaged power are observed. The mode selection as well as the power drops can be predicted by a rate equations model for the longitudinal modes involved. The theoretical explanation of the power drops given in this paper contradicts the previous explanation given in [4].

Close to an averaged power drop, the averaged noise is found to be the strongest, due to mode jumps induced by variations in the external cavity length. We have shown that the region of strong mode jumping can be avoided by setting the external cavity length away from the lowest fractional multiples of the internal cavity length, i.e., $L_{e}=(n+q) L_{i}$, where $n$ is an integer and $q \notin\{0,1 / 2,1 / 3,2 / 3,1 / 4,3 / 4$, etc. $\}$. This observation provides a scientific basis for a procedure often used in practice, in which the distance from the laser to disc is optimized for minimum noise.

\section{REFERENCES}

[1] R. W. Tkach and A. R. Chraplyvy, "Regimes of feedback effects in $1.5-\mu \mathrm{m}$ distributed feedback lasers," J. Lightwave Technol., vol. 4, pp. $1655-1661,1986$.

[2] K. Petermann, Laser Diode Modulation and Noise. Norwell, MA: Kluwer, 1988.
[3] R. Lang and K. Kobayashi, "External optical feedback effects on semiconductor injection laser properties," IEEE J. Quantum Electron., vol. QE-16, pp. 347-355, 1980.

[4] H. Kakiuchida and J. Ohtsubo, "Characteristics of a semiconductor laser with external feedback," IEEE J. Quantum Electron., vol. 30, pp. 2087-2097, 1994

[5] Y. Ikuma and J. Ohtsubo, "Dynamics in a compound cavity semiconductor laser induced by small external-cavity-length change," IEEE J. Quantum Electron., vol. 34, pp. 1240-1246, 1998.

A. S. van de Nes was born in Amsterdam, The Netherlands, in 1977. He received the M.Sc. degree in physics from Leiden University, Leiden, The Netherlands, in 2001, where he studied the behavior of semiconductor lasers. He is currently working toward the Ph.D. degree at Delft University of Technology, Delft, The Netherlands, researching high-density optical recording.

J. J. H. B. Schleipen received the Ph.D. degree in physics from the University of Nijmegen, Nijmegen, The Netherlands, in 1992. His Ph.D. research involved laser spectroscopy and molecular collision dynamicss.

In 1992, he joined Philips Research Laboratories, Eindhoven, The Netherlands, where he has worked on various topics, such as optical feedback in a semiconductor laser, laser scanning and the realization of an all-optical laser neural network. Currently, his work is related to next-generation optical recording concepts.

G. W. 't Hooft received the Ph.D. degree in physics from the University of Leiden, Leiden, The Netherlands, in 1978.

In 1979, he joined Philips Research Laboratories, Eindhoven, The Netherlands, where he has since worked on various topics such as transport and luminescence properties of III-V compounds, the development of (ultra-) fast lasers, and time-resolved optoelectronic characterization methods. His more recent research includes optical mammography and lasing of polymers. He also holds a part-time professorship at the University of Leiden.

M. P. van Exter was born in The Netherlands in 1961. He received the Ph.D. degree in experimental physics in 1988 from the University of Amsterdam, Amsterdam, The Netherlands, where he worked on time-resolved stimulated Raman spectroscopy and the dynamics of surface plasmons.

From 1988 to 1989 , he was with the IBM Watson Research, Yorktown Heights, NY, working on the production of and spectroscopy with terahertz radiation. Since 1989, he has been with Leiden University, working on various types of microcavity lasers, (excess) quantum noise, and vertical cavity surface emitting lasers. His current interests are a.o. quantum information, optical entanglement, and optical chaos (in stable and unstable cavities). 